\title{
Ceftaroline-Induced Agranulocytosis
}

\author{
Najmus Sahar ${ }^{\mathrm{a}, \mathrm{c}}$, Theresa Ratajczak ${ }^{\mathrm{a}}$, Nicholas G. Conger ${ }^{\mathrm{b}}$
}

\begin{abstract}
Ceftaroline fosamil is a new fifth generation cephalosporin with broad-spectrum bacteriocidal activity. It is currently approved for acute bacterial skin and skin structure infections (ABSSSI) and community-acquired bacterial pneumonia (CABP). We report a case of agranulocytosis as a side effect of ceftaroline use, of which the internist needs to be aware.
\end{abstract}

Keywords: Ceftaroline; Agranulocytosis; Neutropenia

\section{Introduction}

Ceftaroline fosamil (Teflaro) has bactericidal activity against methicillin resistant Staphylococcus (MRSA) infections, multidrug resistant strains of Streptococcus pneumoniae, as well as many gram-negative organisms. We present a case of MRSA infective endocarditis in a 24-year-old male, intravenous (IV) drug user who was treated with ceftaroline 600 mg IV every $8 \mathrm{~h}$ for a planned 6 week course. The patient developed leukopenia and agranulocytosis, noted on ceftaroline day 41 that gradually trended back to normal after stopping ceftaroline therapy. Agranulocytosis is a rare but serious side effect associated with beta lactam antibiotics use in large doses for longer than ten days, reported in $<1 \%$ of users. In our patient, the high dose, prolonged treatment course and the timeline are consistent with ceftaroline associated agranulocytosis. Monitoring weekly complete blood count and patient education about neutropenia symptoms are important aspects of outpatient parenteral antibiotic therapy

Manuscript accepted for publication April 18, 2016

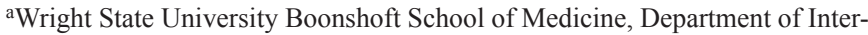
nal Medicine, Dayton, OH, USA

${ }^{b}$ Wright-Patterson Medical Center, Wright State University Boonshoft School of Medicine, Department of Internal Medicine, Dayton, OH, USA

${ }^{\mathrm{c} C}$ Corresponding Author: Najmus Sahar, Wright State University Boonshoft School of Medicine, Department of Internal Medicine, Dayton, OH, USA. Email: drnajum83@yahoo.com

doi: http://dx.doi.org/10.14740/jmc2489w with ceftaroline.

\section{Case Report}

A 24-year-old Caucasian male presented to the emergency department with right-sided chest pain of 3 days duration. He reported a constant, dull chest pain radiating to the left side. The pain was associated with chills and a productive cough with blood tinged sputum. Fever, night sweats, recent antibiotic use, travel, or hospitalization were not reported. The review of systems was positive for fatigue, shortness of breath, abdominal pain, non-bloody diarrhea, and a bilateral lower extremity rash. The patient had no significant past medical or surgical history. Social history included a 16 pack year tobacco use, prior heavy alcohol intake, and current IV heroin abuse. The patient had no known medical allergies and was not taking any prescribed or over-the-counter medications.

Physical examination revealed an ill-appearing, young male with multiple tattoos. He was afebrile and hemodynamically stable, saturating well on room air. Cardiac examination revealed tachycardia with a heart rate of 143 beats/min and a grade III/VI systolic murmur along the left sternal border. No jugular venous distention or lower extremity edema was noted. Macular rash was noted on bilateral lower extremities from the knees down.

Admission laboratory workup showed elevated cardiac enzymes with troponin I of $0.01 \mathrm{ng} / \mathrm{mL}$ (reference range 0.00 - $0.07 \mathrm{ng} / \mathrm{mL}$ ). Leukocyte count was $11,700 / \mathrm{mm}^{3}$ (reference range $3,800-10,800 / \mathrm{mm}^{3}$ ) with $81 \%$ neutrophils, $11 \%$ lymphocytes, $4 \%$ monocytes, and 4\% bands, hemoglobin of 12.7 $\mathrm{g} / \mathrm{dL}$ (reference range $13.8-17.2 \mathrm{~g} / \mathrm{dL}$ ), and platelet count of $64,000 / \mathrm{mm}^{3}$ (reference range $130,000-400,000 / \mathrm{mm}^{3}$ ). The comprehensive metabolic panel was significant for a serum sodium concentration of $128 \mathrm{mEq} / \mathrm{L}$, albumin of $2.8 \mathrm{~g} / \mathrm{dL}$, aspart aminotransferase (SGOT) of $115 \mathrm{U} / \mathrm{L}$, and alanine aminotransferase (SGPT) of $75 \mathrm{U} / \mathrm{L}$. C- reactive protein (CRP) was 16.5 $\mathrm{mg} / \mathrm{dL}$, haptoglobin $241 \mathrm{mg} / \mathrm{dL}$ and LDH $226 \mathrm{U} / \mathrm{L}$.

EKG showed sinus tachycardia with non-specific ST-T wave changes. Chest radiograph demonstrated bilateral pulmonary infiltrates and possible septic emboli. Subsequent CT chest showed multiple non-calcified cavitary pulmonary nodules throughout both lungs compatible with septic emboli, and a moderate size right pneumothorax. CT abdomen showed 
Table 1. Patients Absolute Neutrophil and White Blood Cell Count During and After Ceftaroline Treatment

\begin{tabular}{|c|c|c|c|}
\hline Date & $\begin{array}{l}\text { WBC count } \\
\text { in }\left(10^{3} / \mathrm{mm}^{3}\right)\end{array}$ & $\begin{array}{l}\text { Absolute neutrophil } \\
\text { count (cells } / \mu \mathrm{L})\end{array}$ & \\
\hline June 28, 2015 & 11.7 & 9,945 & \\
\hline July 03, 2015 & 5.0 & 3,564 & \\
\hline July 04, 2015 & 6.6 & 4,884 & \\
\hline August 09, 2015 & 1.5 & 0 & \\
\hline August 11, 2015 & 1.7 & 0 & \\
\hline August 12, 2015 & 3.3 & 858 & \\
\hline August 15, 2015 & 11.2 & 6,608 & \\
\hline
\end{tabular}

splenomegaly, gallbladder wall thickening, and a possible pericholecystitic fluid.

Blood cultures were obtained, and empiric treatment of severe sepsis, due to suspected infective endocarditis and acute cholecystitis, was initiated with vancomycin, pipperacilin-tazobactam, and levofloxacin. A chest tube was placed in the emergency room, and the patient was admitted for further management. Transthoracic echocardiogram showed tricuspid valve vegetations with severe tricuspid regurgitation and an ejection fraction of $40 \%$, establishing the diagnosis of infective endocarditis. Cardiology and cardiothoracic surgery were consulted and recommended aggressive antibiotic treatment without any urgent need for surgical intervention. Further laboratory workup was positive for hepatitis $\mathrm{C} \mathrm{Ab}$ with undetectable viral load and negative for HIV, RPR, hepatitis B, ANA, anti-DS DNA and RF Ab.

Blood cultures grew gram-positive cocci, reported as MRSA with susceptibilities to vancomycin, minimum inhibitory concentration (MIC) of $1 \mathrm{mg} / \mathrm{L}$, daptomycin $\mathrm{MIC}$ of 0.5 $\mathrm{mg} / \mathrm{L}$, and ceftaroline $\mathrm{MIC}$ of $0.5 \mathrm{mg} / \mathrm{L}$. Antibiotic regimen was narrowed to ceftaroline $600 \mathrm{mg}$ twice daily and daptomycin $500 \mathrm{mg}$ daily on day 2 . In addition, a 5 day course of metronidazole was added for coexisting cholecystitis. Blood cultures became sterile on day 3. Daptomycin was discontinued on day 7 , and IV ceftaroline monotherapy was continued with $600 \mathrm{mg}$ every $8 \mathrm{~h}$ for 6 weeks total. The patient was discharged to a rehabilitation facility on ceftaroline day 7 with leukocyte count of $6,400 / \mathrm{mm}^{3}$ and ANC of 4,928 cells $/ \mu \mathrm{L}$, to complete the course of his IV antibiotic.

One day after discharge from the rehabilitation facility on ceftaroline day 41, the patient presented to the hospital with generalized abdominal pain of 1-day duration. Physical examination revealed a febrile male with generalized abdominal tenderness without rebound or guarding. Readmission laboratory workup showed leukocyte count of $500 / \mathrm{mm}^{3}$ and ANC of 0 , hemoglobin $12.1 \mathrm{~g} / \mathrm{dL}$, hematocrit of $36.5 \%$, and platelet count of $128,000 / \mathrm{mm}^{3}$. CT abdomen showed inflammation of small bowl loops and resolution of cavitary pulmonary nodules. The patient was started on aztreonam and daptomycin for presumed neutropenic fever but discontinued after $48 \mathrm{~h}$, due to concern for a drug-induced fever. Blood cultures remained negative. Leukocyte count gradually trended back to normal and reached $3,300 / \mathrm{mm}^{3}$ four days later and $10,300 / \mathrm{mm}^{3}$ eight days after stopping ceftaroline therapy (Table 1).

\section{Discussion}

High dose IV beta-lactam antibiotics, such as penicillins and cephalosporins, have been used for decades to treat numerous bacterial infections. Cephalosporins have been used more than $40 \%$ of the time as outpatient parenteral therapy due to broad-spectrum coverage and known safety profile [1]. With the emergence of new multidrug-resistant pathogens, there is a constant need for more effective, extended spectrum antibiotics with limited side effects.

Ceftaroline fosamil (Teflaro) is a new parenteral cephalosporin with bactericidal activity against resistant gram-positive organisms including MRSA and multidrug-resistant strains of Streptococcus pneumoniae and gram negatives including Enterobacteriaceae [2]. In 2010, the FDA originally approved ceftaroline fosamil for ABSSSI and CABP. The recommended dosage is $600 \mathrm{mg}$ every $12 \mathrm{~h}$ for 5 - 14 days with dose adjustment indicated in patients with creatinine clearance $(\mathrm{CrCl})<$ $50 \mathrm{~mL} / \mathrm{min}$, including end-stage renal disease (ESRD) and dialysis patients [3].

Ceftaroline is well tolerated in general. Common adverse reactions include diarrhea, nausea, and rash in more than $2 \%$ of patients. Hypersensitivity occurred in $0.3 \%$, and direct combs test seroconversion without hemolysis has been observed in $10.8 \%$ in pooled phase 3 clinical trials [3]. Off-label utility of ceftaroline for other systemic MRSA-related infections over time has shown improved outcome [4], but also brought to light case reports with rare side effects related to this novel agent [5-7].

Neutropenia, defined as absolute neutrophil count $<1,500$ cells $/ \mu \mathrm{L}$ and agranulocytosis $<100$ cells $/ \mu \mathrm{L}$, is an extremely rare but serious side effect associated with beta-lactam antibi- 


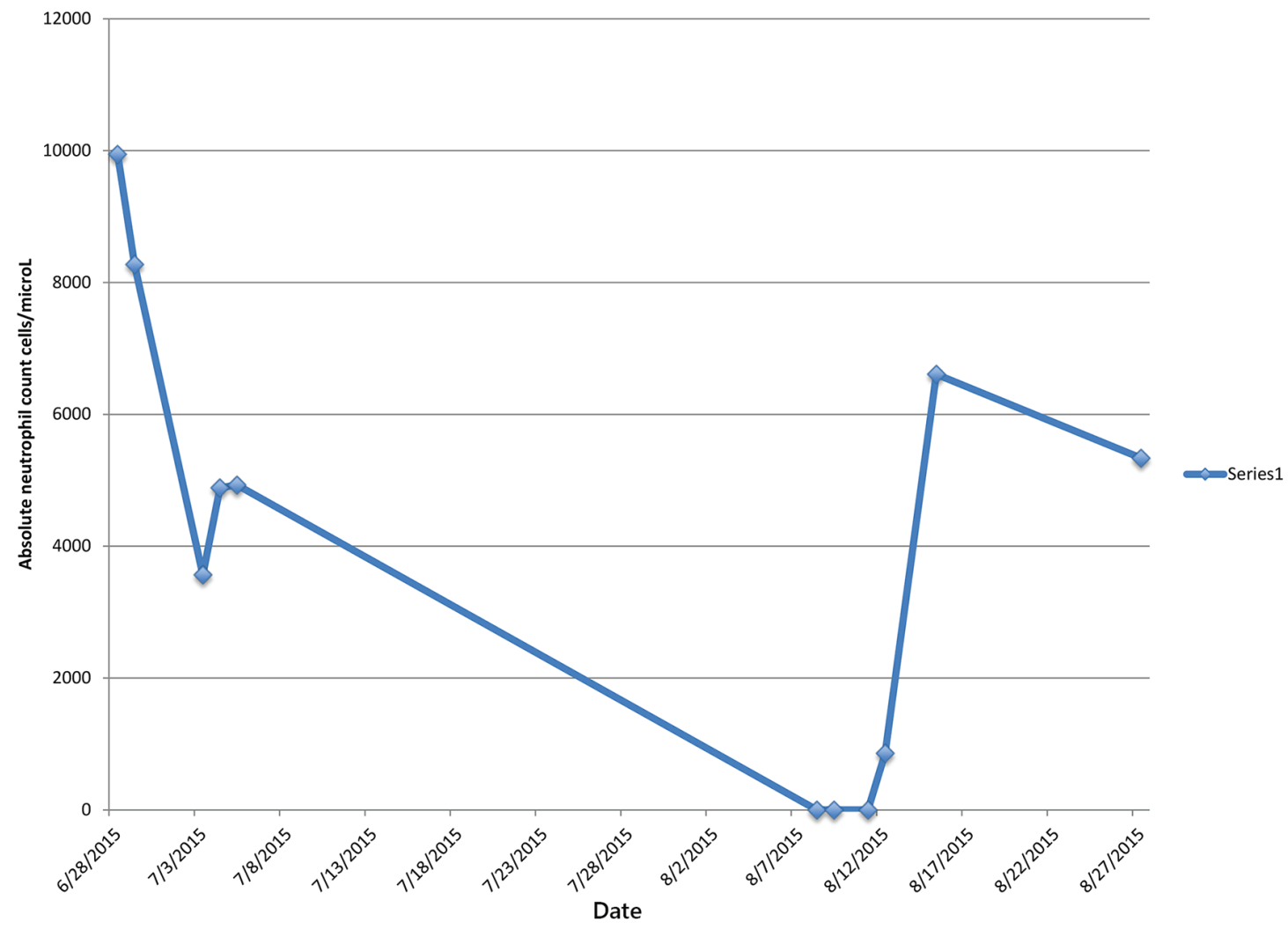

Figure 1. Patient's absolute neutrophil count during and after completion of ceftaroline treatment.

otic use. In our literature search, we found a large number of cases reporting hematologic toxicities associated with cephalosporin use including ceftriaxone, cefuroxime, and cefepime. The incidence rate of neutropenia was less than $1 \%$ with IV cephalosporin therapy longer than 10 days [1], but historically has been reported as high as $5-15 \%$ with large doses and courses longer than 2 weeks [8]. The exact mechanism is unclear, but proposed theories revolve around either immune mediated cell destruction [9] or direct toxic effects of medication on bone marrow precursors in a dose dependent manner $[8,10]$. Complete recovery within 10 days after stopping the offending agent is well documented in most cases [8].

There are few reports on usage and outcomes of ceftaroline for MRSA-related systemic infection. A large retrospective analysis involving 527 patients treated with ceftaroline for various MRSA infections showed $88 \%$ clinical success [11]. In a small retrospective study in 2014 involving 12 patients, ceftaroline was discontinued in $75 \%$ of patients $(9 / 12)$ secondary to adverse events including hematologic and dermatologic reasons [12].

Off-label ceftaroline was selected for our patient due to cost effectiveness and favorable adverse effect profile. More frequent dosage (e.g., every $8 \mathrm{~h}$ ) was used to provide higher target attainment $(\% \mathrm{~T}>\mathrm{MIC})$ based on a prior study [13]. Use of ceftaroline $600 \mathrm{mg}$ every $8 \mathrm{~h}$ for 6 weeks duration is consistent with the increased risk of cephalosporin-induced agranulocytosis based on prior reports $[5,6,14]$.

In our patient, the development of agranulocytosis after 6 weeks of therapy and complete recovery of neutrophil count within 7 days support the fact that neutropenia was drug-induced (Figs. 1 and 2). Workup for secondary causes of agranulocytosis was negative, and the patient was not on any other medication known to cause agranulocytosis. Neutropenia and agranulocytosis in general increase the risk of bacterial and fungal infections, but the incidence is low with drug-induced neutropenia. Upon readmission, the patient was afebrile and had neither signs nor symptoms of infection.

Patients with leukopenia on presentation are at higher risk for developing drug-induced neutropenia and agranulocytosis that can lead to increased morbidity and mortality. Use of betalactam antibiotics including ceftaroline in higher doses or for longer duration requires patient education regarding the signs and symptoms of the side effects. Close monitoring with weekly $\mathrm{CBC}$ and discontinuation of the offending agent is recommended if hematologic toxicity is suspected [1].

Due to limited adverse effects, favorable dosage, and broad coverage against MRSA and resistant infections, ceftaroline utilization will increase with time and expose the impact of this new drug on patient outcomes. Our case was the third case report of agranulocytosis associated with ceftaroline use.

\section{Conclusion}

Neutropenia and agranulocytosis are extremely rare side effects associated with large doses and longer duration of beta- 


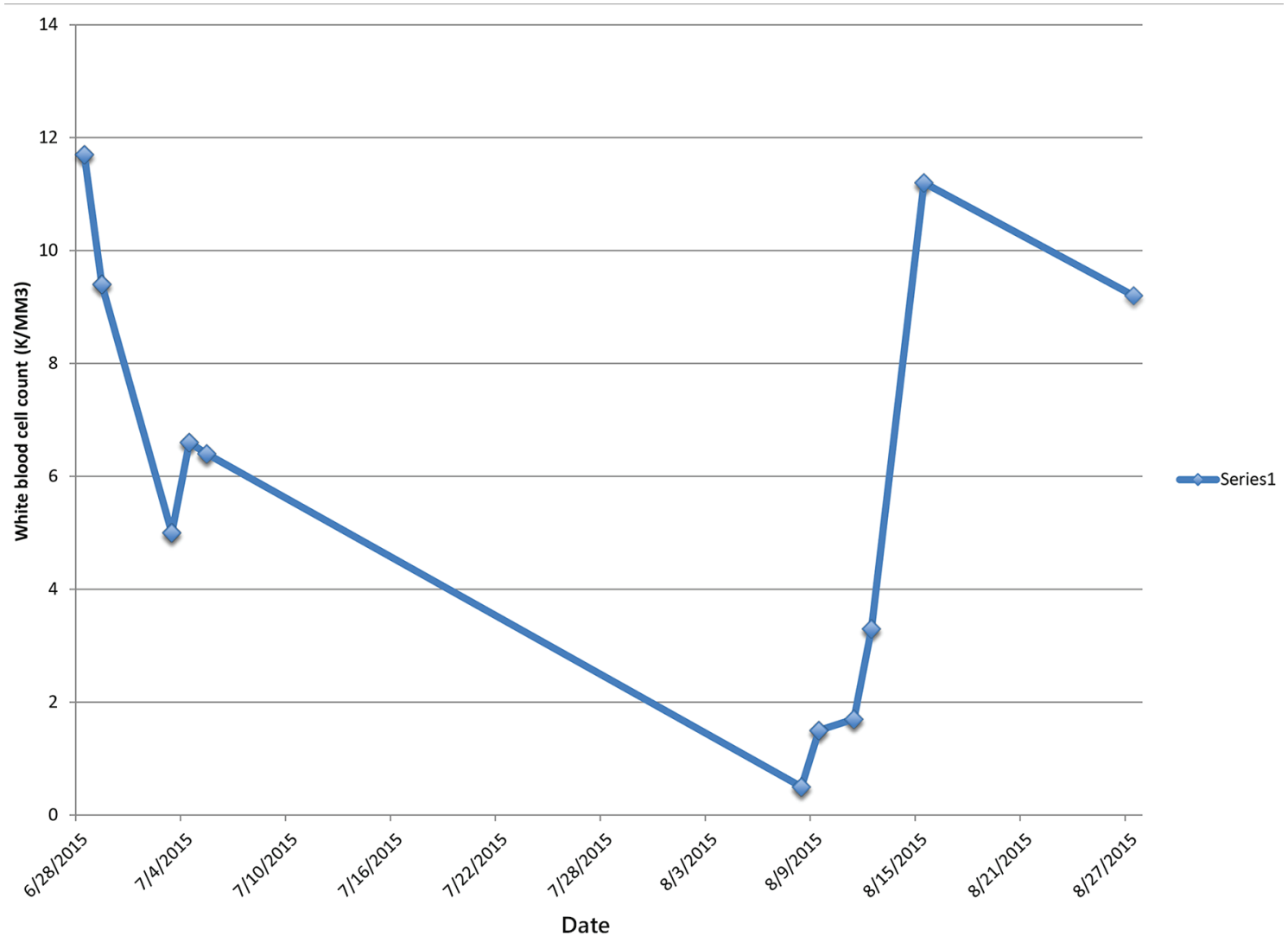

Figure 2. Patient's white blood cell count during and after completion of ceftaroline treatment.

lactam antibiotics use, and ceftaroline is no exception. Close monitoring of $\mathrm{CBC}$ and patient education are the cornerstones for avoiding unnecessary complications.

\section{Acknowledgments}

We would like to acknowledge the assistance of Dr. Ronald J. Markert, Ph.D., Vice Chairman for Research, Department of Internal Medicine, Wright State University Boonshoft School of Medicine, Dayton Ohio, for his comments and critical review of the manuscript.

\section{References}

1. Tice AD, Rehm SJ, Dalovisio JR, Bradley JS, Martinelli LP, Graham DR, Gainer RB, et al. Practice guidelines for outpatient parenteral antimicrobial therapy. IDSA guidelines. Clin Infect Dis. 2004;38(12):1651-1672.

2. Pfaller MA, Flamm RK, Sader HS, Jones RN. Ceftaroline activity against bacterial organisms isolated from acute bacterial skin and skin structure infections in United States medical centers (2009-2011). Diagn Microbiol Infect Dis. 2014;78(4):422-428.

3. Teflaro (ceftaroline fosamil) package insert. Teramo, Italy: Forest Laboratories; 2012.
4. Arshad S, Hartman P, Zervos MJ. A novel treatment option for MRSA pneumonia: ceftaroline fosamil-yielding new hope in the fight against a persistent infection. Expert Rev Anti Infect Ther. 2014;12(7):727-729.

5. Rimawi RH, Frenkel A, Cook PP. Ceftaroline - a cause for neutropenia. J Clin Pharm Ther. 2013;38(4):330-332.

6. Yam FK, Kwan BK. A case of profound neutropenia and agranulocytosis associated with off-label use of ceftaroline. Am J Health Syst Pharm. 2014;71(17):1457-1461.

7. Griffiths CL, Gutierrez KC, Pitt RD, Lovell RD. Eosinophilic pneumonia induced by ceftaroline. Am J Health Syst Pharm. 2014;71(5):403-406.

8. Neftel KA, Hauser SP, Muller MR. Inhibition of granulopoiesis in vivo and in vitro by beta-lactam antibiotics. J Infect Dis. 1985;152(1):90-98.

9. Murphy MF, Metcalfe P, Grint PC, Green AR, Knowles $\mathrm{S}$, Amess JA, Waters AH. Cephalosporin-induced immune neutropenia. Br J Haematol. 1985;59(1):9-14.

10. Neftel KA, Hauser SP, Muller MR, Walti M. Cephalosporin-induced neutropenia. Br J Haematol. 1986;62(2):394397.

11. Casapao AM, Davis SL, Barr VO, Klinker KP, Goff DA, Barber KE, Kaye KS, et al. Large retrospective evaluation of the effectiveness and safety of ceftaroline fosamil therapy. Antimicrob Agents Chemother. 2014;58(5):25412546.

12. Jain R, Chan JD, Rogers L, Dellit TH, Lynch JB, Potting- 
er PS. High incidence of discontinuations due to adverse events in patients treated with ceftaroline. Pharmacotherapy. 2014;34(7):758-763.

13. Vidaillac C, Leonard SN, Rybak MJ. In vitro activity of ceftaroline against methicillin-resistant Staphylococcus aureus and heterogeneous vancomycin-intermediate
S. aureus in a hollow fiber model. Antimicrob Agents Chemother. 2009;53(11):4712-4717.

14. LaVie KW, Anderson SW, O'Neal HR, Jr., Rice TW, Saavedra TC, O'Neal CS. Neutropenia Associated with Long-Term Ceftaroline Use. Antimicrob Agents Chemother. 2015;60(1):264-269. 